\title{
Technology-Assisted Psychosocial Interventions for Childhood, Adolescent, and Young Adult Cancer Survivors: A Systematic Review and Meta-Analysis
}

\author{
Anao Zhang, PhD, MSW, ${ }^{1, *}$ Bradley Zebrack, PhD, MSW, MPH, ${ }^{1}$ Chiara Acquati, PhD, MSW, MA, ${ }^{2}$ \\ Michael Roth, MD, ${ }^{3}$ Nina Jackson Levin, MSW, ${ }^{4}$ Kaipeng Wang, $\mathrm{PhD},{ }^{5}$ and Samantha Schwartz, MSW ${ }^{1}$
}

\begin{abstract}
Purpose: Technology-assisted interventions are essential in supporting cancer survivors' psychosocial outcomes, especially for childhood, adolescent, and young adult (AYA) cancer survivors, a tech-savvy generation. This study aims to systematically evaluate review and meta-analyze technology-assisted interventions for childhood and AYA cancer survivors.
\end{abstract}

Methods: Following the Preferred Reporting Items for Systematic Review and Meta-Analysis (PRISMA) guidelines, the study team used a pre-set of key words and searched studies across 11 electronic databases and 4 professional websites, and conducted a manual search of reference lists from published reviews. Meta-analysis of small sample size corrected Hedges' $g$ was conducted using meta-regression with robust variance estimation. Results: Final analysis included a total of 28 clinical trials, including 237 effect sizes reported an overall statistically significant treatment effect of technology-assisted psychosocial interventions for childhood and AYA cancer survivors, $g=0.382,95 \%$ confidence interval (CI) 0.243 to $0.521, p<0.0001$. Subgroup analysis revealed that distraction-based interventions and interventions for psychosocial and emotional health were overall statistically significant, whereas interventions for childhood and AYA cancer survivors' cancer knowledge outcomes and physical and functional health outcomes were statistically nonsignificant. Moderator analysis found intervention target was a significant moderator.

Conclusions: Technology-assisted interventions for childhood and AYA cancer survivors were overall effective across domains of survivorship outcomes. Favorable evidence was found primarily for childhood cancer survivors with limited support for AYA cancer survivors.

Implications for Cancer Survivors: Although existing technology-assisted interventions are overall promising, research support for cancer survivors from different age groups and with different psychosocial challenges varies and should be considered individually.

Keywords: technology-assisted intervention, childhood and AYA cancer, survivorship outcomes, meta-analysis

\section{Introduction}

C ANCER IS THE LEADING cause of disease-related death past infancy among children and adolescents and young adults (AYAs) in the United States and across the globe. ${ }^{1,2}$ In the United States, $\sim 11,000$ and 89,500 new cancer diagnoses were estimated for children (0-14 years of age) and
AYA patients (15-39 years of age) in 2020, respectively. ${ }^{2,3}$ Advances in the therapeutic and supportive care have improved the overall 5-year cancer survival rate among children and AYAs to $80 \%$ or higher, ${ }^{4,5}$ which has resulted in a large and growing population of childhood and AYA cancer survivors. ${ }^{6,7}$ As the prognosis for childhood and AYA cancer patients continues to improve, increased attention has been

\footnotetext{
${ }^{1}$ University of Michigan School of Social Work, Ann Arbor, Michigan, USA.

${ }^{2}$ University of Houston Graduate College of Social Work; and The University of Texas MD Anderson Cancer Center Department of Health Disparities Research, Houston, Texas, USA.

${ }^{3}$ Department of Pediatrics, The University of Texas MD Anderson Cancer Center, Houston, Texas, USA.

${ }^{4}$ University of Michigan School of Social Work, and Department of Anthropology, Ann Arbor, Michigan, USA.

${ }^{5}$ Graduate School of Social Work, University of Denver, Denver, Colorado, USA.

*ORCID ID (https://orcid.org/0000-0002-3199-1113).
} 
focused on the health-related quality of life (HRQoL), including psychosocial, behavioral, and functional health, among members of this population. ${ }^{8,9}$

Indeed, studies have reported that over $60 \%$ of childhood and AYA cancer survivors experience adverse psychosocial, behavioral, and functional health outcomes that compromise their HRQoL for many years after completion of cancer treatment. ${ }^{10}$ Key challenges affecting survivors' HRQoL include the following: (1) distressing experiences during active invasive cancer treatment (e.g., fear, pain, and nausea $)^{11,12}$; (2) psychosocial and emotional challenges both during and after treatment, including psychological distress, fear of recurrence, infertility, and reproductive/sexual health challenges $^{13-15}$; (3) limited knowledge about and engagement in survivorship care ${ }^{16-18}$; and (4) impaired physical and functional health due to adverse and late effects of cancer treatment. $^{19-21}$ If left unaddressed, these challenges have been found to be associated with childhood and AYA cancer survivors' disengagement from survivorship care, reduced self-efficacy, increased risk health behaviors, including suicide, and compromised HRQoL. ${ }^{19,22-26}$

While effective interventions are available for childhood and AYA cancer survivors' psychosocial, behavioral, and functional health, ${ }^{27,28}$ these interventions often fail to reach the population in need. First, there is a significant psychooncology workforce shortage supporting childhood and AYA cancer survivors, ${ }^{29,30}$ resulting in many childhood and AYA survivors not having access to essential psycho-oncology care such as depression treatment or functional rehabilitation. Second, even for childhood and AYA survivors who have access to psycho-oncology services, high proportions of these patients drop out of services, and compared with their younger and older counterparts, AYA survivors are the most likely to disengage from supportive cancer care due to their unique developmental and cancer treatment features, that is, the transition of their care from pediatric to medical oncology. ${ }^{31,32}$

Given the above-mentioned challenges, technologyassisted psychosocial interventions have received increased attention among psycho-oncology practitioners and researchers as the result of several major strengths. In this article, due to a lack of agreed-upon typology/taxonomy of technologyassisted health interventions, ${ }^{33}$ we broadly define technologyassisted interventions as any type of intervention that involves the use of technology, ranging from text messages and wearable devices to computers and virtual reality devices. We define psychosocial interventions broadly as any type of intervention that is not solely medical and/or pharmacological, for example, psychotherapeutic interventions, psychoeducational interventions, behavioral skills training, and peer support groups. Technology-assisted psychosocial interventions have important potential in reducing barriers to access, which can allow services to be delivered at patients' preferred location and time. ${ }^{34,35}$ More importantly, as members of a tech-prone and tech-savvy generation, childhood and AYA cancer survivors are more likely to engage in technologyassisted psychosocial interventions, as many of these interventions are delivered in their preferred methods of communication (e.g., social media-based peer support groups or text messages to improve treatment compliance). ${ }^{36,37}$ Finally, recent advances in machine learning and artificial intelligence have enabled key innovations in the delivery of psycho-oncology care for childhood and AYA cancer survi- vors. For example, psycho-oncology researchers have started to explore the use of machine learning models to inform AYA cancer survivors' survivorship care planning. ${ }^{38}$

Considering the major strengths and important potential of technology-assisted psychosocial interventions for childhood and AYA cancer survivors across cancer centers and geographic locations, it is critical to systematically evaluate technology-assisted psychosocial interventions to guide the field of psycho-oncology and inform future research on survivorship care. To our knowledge, three relevant reviews have been conducted to date: two narrative systematic reviews of digital health (or mHealth) interventions, one for childhood and the other for AYA cancer survivors, ${ }^{39,40}$ and one meta-analysis focusing on distance-delivered physical activity interventions for childhood cancer survivors. ${ }^{28}$ The two review articles ${ }^{39,40}$ concluded that technology-assisted interventions are acceptable to and feasible for pediatric and AYA cancer survivors in general and called for future research to focus on formally evaluating the efficacy of technology-assisted interventions. In addition, Mizrahi et al. $^{28}$ meta-analyzed four clinical trials of distancedelivered physical activity interventions for childhood cancer survivors and found preliminary evidence supporting their benefits for physical function and psychological outcomes, but not for physical activity and physiological outcomes. These three highly valuable studies consistently called for investigators to do the following: (1) conduct a meta-analysis to formally evaluate the efficacy of technology-assisted interventions for pediatric and AYA survivors; (2) include various technology-assisted interventions targeting various outcome domains, as they often interact with each other and coexist; and (3) evaluate key moderators, if data allow, such as cancer survivors' age or race in relation to treatment effect size, which will deepen the understanding of whether certain interventions are better tailored to respond to the unique needs of specific patient populations.

In response to key gaps in the literature, this work reports on the systematic review and meta-analysis of technologyassisted psychosocial interventions for childhood and AYA cancer survivors, including multiple domains of survivorship outcomes (defined later in the inclusion criteria). In this review, a person is considered a cancer survivor from the time of diagnosis until the end of life, that is, it includes childhood and AYA cancer patients both during and after cancer treatment. $^{41}$ Our primary hypothesis is that technologyassisted psychosocial interventions are effective overall for childhood and AYA cancer survivors across multiple survivorship outcome domains. We also examine whether the interventions are effective for specific outcome domains when evaluated separately. Finally, we explore whether any important study, intervention, or participant characteristics (e.g., age, race, treatment length, and study design) moderates the treatment effect size of technology-assisted interventions.

\section{Methods}

This study was conducted following Preferred Reporting Items for Systematic Review and Meta-Analysis (PRISMA) guidelines, ${ }^{42}$ by an investigative team equipped with expertise in pediatric and AYA psychosocial oncology, cancer survivorship, and procedures for conducting systematic review and meta-analysis. 


\section{Search procedure and study eligibility}

The study team performed a systematic search of controlled clinical trials (with or without random participant assignment) that evaluated technology-assisted psychosocial interventions for pediatric and/or AYA cancer survivors. Specifically, 11 electronic databases, 4 professional websites, and a manual search of reference lists from published reviews (30 systematic reviews and 6 meta-analysis studies) were used to generate an initial pool of studies for screening (Supplementary Appendix SA1). To identify studies focusing on childhood and AYA cancer survivors, we searched using the following key terms: "childhood cancer" or "pediatric cancer" or "childhood oncology" or "pediatric oncology" or "adolescent and young adult" or "AYA" or "young adult cancer" or "young adult oncology" or "youth cancer" or "youth oncology." To identify studies that evaluated psychosocial interventions, we used the key terms "psychological" or "social" or "psycho*" or "behavior*", or "support*" or "education*" or "training."

All studies published in English were searched from inception to August 1, 2020 (the date the initial search was completed). To be eligible for inclusion, a study needed to (1) be a controlled trial (with or without randomization); (2) evaluate one or more psychosocial intervention(s); (3) target pediatric, adolescent, and/or young adult cancer survivors (i.e., individuals 0-39 years of age with a cancer diagnosis); and (4) utilize technology in the delivery of the intervention, including those that used technology as the primary mode of treatment delivery (e.g., virtual reality goggles) and those that used technology in an assistive role (e.g., text messaging). The study could target any outcome in childhood and AYA cancer survivors, ranging from physical (e.g., pain and tremor) and psychological (e.g., depression and distress) outcomes to quality of life, cancer knowledge, and social relations (e.g., self-isolation) outcomes. Studies were excluded only if they evaluated medical or pharmaceutical treatment, there was no comparison/control group in the study, or there was no outcome data on intervention efficacy or effectiveness.

\section{Data extraction}

A data extraction sheet was pre-developed by the study team to systematically code and extract information for all included studies (available upon request from the first author). Two research assistants independently screened all studies first by reading study titles and abstracts, and then by reading the full text of the remaining articles using Covidence, a Cochrane-recommended screening platform. Disagreements between the two research assistants were resolved by consensus first, and, if not successful, through discussion with a third/senior study team member. Interscreener reliability was $92 \%$ and inter-reviewer reliability was $89 \%$, both of which indicated satisfactory reliability.

\section{Publication bias and quality of study assessment}

Publication bias was assessed using the Cochrane Collaboration's Risk of Bias Tool, version 2 (RoB 2), ${ }^{43}$ for randomized controlled trials, and the Risk of Bias in Nonrandomized Studies - of Interventions (ROBINS-I) tool for controlled trials without randomization. ${ }^{44}$ Both tools evalu- ate key aspects/domains of clinical trial studies that are subject to research bias, such as the randomization process, the handling of missing data, and the selective reporting of data. Each study was evaluated using a three-category response: "+" = low risk of bias, "?"= unsure/moderate risk of bias, or " $x$ " $=$ serious or critical risk of bias. Publication bias was assessed visually using a funnel plot and statistically using Vevea and Woods' sensitivity analysis using a priori weight functions. ${ }^{45}$ A reasonably symmetric funnel plot-in which both statistically significant and insignificant effect sizes are included and analyzed-is a sign of no publication bias. Vevea and Woods' sensitivity analysis calculates an observed effect size and a theoretical effect size assuming the funnel plot is symmetric (i.e., there is no publication bias). If the distance between the two lines is not statistically significant, it can be viewed as supporting no publication bias.

\section{Meta-analytic procedures}

All trial outcomes were continuous variables and small sample size corrected Hedges' $g$ was calculated to reflect standardized mean difference effect size. ${ }^{46}$ The sampling variance of Hedges' $g$ was obtained as a function of the $g$ and its associated sample size. ${ }^{46}$ Meta-analysis was conducted using meta-regression with robust variance estimation (RVE). ${ }^{47}$ Meta-regression with RVE was chosen because of the clustered nature of the data. Specifically, multiple effect sizes of the same construct were reported in a single study, which created dependence among those effect sizes from the same study. Meta-regression with RVE effectively handles the dependence among effect sizes from the same study without excluding these effect sizes. ${ }^{47,48}$ Furthermore, metaregression with RVE produces unbiased estimates of an overall treatment effect size that is robust across different variance assumptions, that is, robust for both fixed- and random-effects models. In addition to estimating an overall treatment effect size, we also planned subgroup analyses and moderator analyses based on important intervention and patient characteristics, for example, the role of technology, the treatment modality, and patients' age.

Specifically for moderator analyses, we conducted univariate meta-regression analysis with the following: (1) key patient demographic variables (i.e., cancer patients' age, sex, racial background, treatment stage, and current treatment received, if applicable); (2) key study design variables, that is, randomization (random vs. nonrandom assignment), type of comparison (e.g., active treatment vs. treatment-as-usual vs. waitlist or attention control); and (3) intervention characteristics, that is, intervention modality, targeted outcome, nature of intervention (e.g., psychotherapeutic vs. supportive intervention), role of technology, and human clinician involvement. One caveat in understanding results from metaregression with RVE is that when a statistic has a degree of freedom lower than 4 , a stricter $p$ value (i.e., $p<0.01$ ) should be used for interpreting statistical significance. Due to the fact that during the course of this study, we conducted multiple statistical tests using the same data and thus needed to adjust for an inflated Type I statistical error, in this article, we consider a $p$-value of 0.01 as being statistically significant for all result interpretations. Sensitivity analyses were conducted to account for risk of bias and outliers to ensure that 
findings did not significantly change. All data analyses were conducted using R Statistical Software (version 4.0.3) with the "metaphor" and "robumeta" packages.

\section{Results}

\section{Search results and study characteristics}

A total of 10,026 records were retrieved from electronic database and manual searches after duplications were removed. Title and abstract screening excluded 9932 studies that clearly did not meet inclusion criteria, leaving 94 studies for full text eligibility screening. Sixty-six studies were further excluded for various reasons (see Fig. 1 notes), resulting in a final analytical sample size of 28 clinical trials.

The 28 trials included 237 effect size estimates and a total sample size of 2006 childhood and AYA survivors (Table 1). Trials were published between 1987 and 2019, including 1 published in 1987, 6 published between 2000 and 2010, 12 published between 2011 and 2015, and 9 published between 2016 and 2019. Participants' average age ranged from 9 to 32 years, with 16 studies (109 effect sizes) focusing on childhood cancer survivors, 9 studies (100 effect sizes) focusing on AYA cancer survivors, and 3 studies (28 effect sizes) focusing on both. In the trials that reported patients' sex $(n=27), 47 \%$ of the patients were female $(n=920)$, and 25 studies reported a total of 902 Caucasian participants (55.66\%). Twenty-two out of 28 trials (78.6\%) included childhood and AYA cancer survivors with different cancer diagnoses, leaving 6 trials that included childhood and AYA cancer survivors with only one diagnosis. All but two studies were randomized controlled trials and two were controlled trials without randomization.

Most clinical trials (15 studies, including 89 effect sizes) reported psychosocial and emotional health outcomes among childhood and AYA cancer survivors. Five studies (25 effect sizes) reported the outcome of distracting childhood and AYA survivors from invasive cancer treatment, leaving 6 studies ( 80 effect sizes) and 8 studies (43 effect sizes) that focused on the outcomes of cancer-related knowledge and functional health among childhood and AYA survivors, respectively.

Over $80 \%$ of the studies $(n=22)$ used technology as the primary intervention or in the venue of the intervention delivery, while 6 studies used technology as an assistive tool for intervention, for example, use of text messages for between-session check-ins. Over half of the interventions ( $n=19$, or $67.9 \%$ ) were individual-based interventions and the remainder were group-based interventions. Thirteen studies involved a human clinician as the primary provider, while 15 studies involved a human clinician (in an assistive role) for logistic or administrative check-in purposes. Various technologies were used across studies, such as tablets, cellphones, computers/laptops, Fitbits/wearables, and virtual reality devices (e.g., goggles), among others.

\section{Publication bias and risk of bias}

The funnel plot for publication bias (Fig. 2) was reasonably symmetric, which indicated low concern for publication bias. The Vevea and Woods' sensitivity weight model supported the absence of publication bias with the observed overall treatment effect size (red line) not significantly different from a theoretical overall treatment effect size (blue line), assuming the funnel plot is symmetric. Risk of bias assessment (Table 2) identified overall low risk of bias for both the randomized controlled trials and the controlled trials without randomization in the study. Unsure or moderate risk of bias arising from the randomization process and bias due to missing outcome data were identified in several of the trials. No other concern about risk of bias was present.

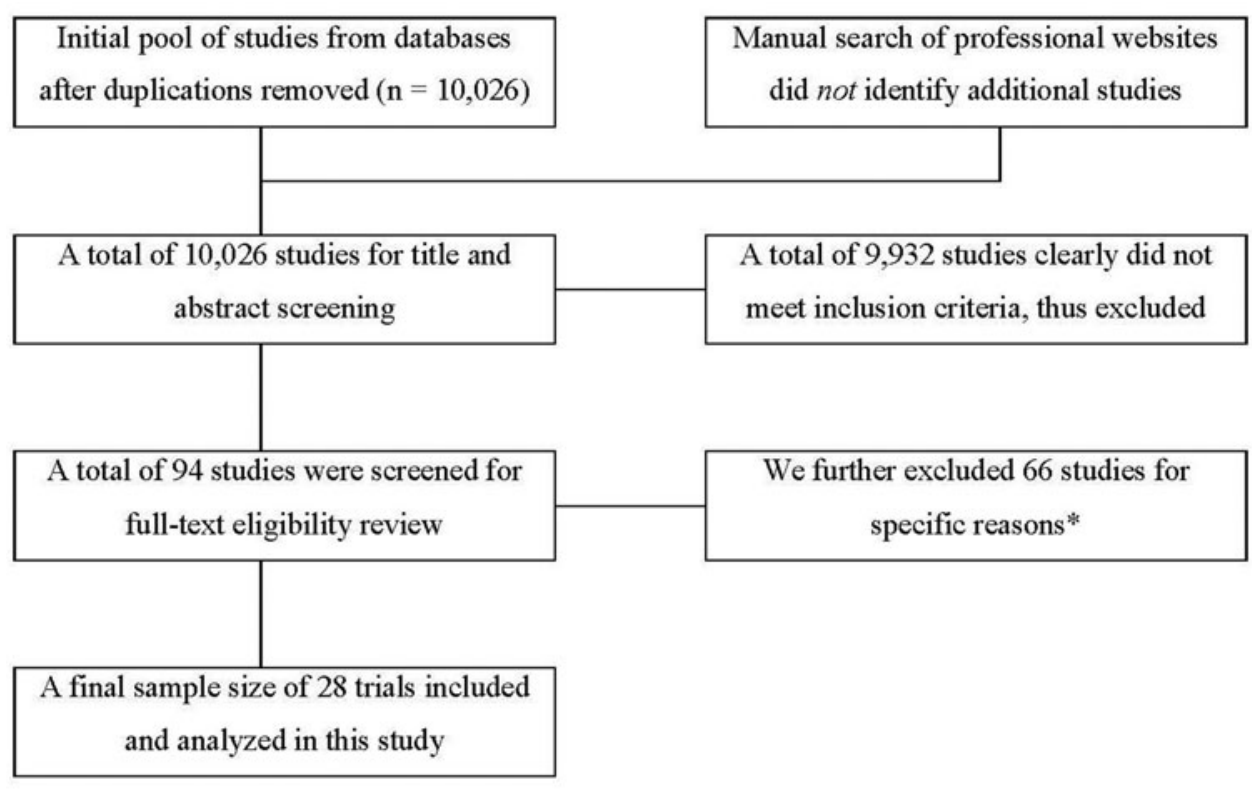

* Reasons for full-text exclusion: 1 . No control group $(n=9) ; 2$. Included patients older than 39 years old $(n=4) ; 3$. Interventions focused on parents and parent related outcomes $(n=7) ; 4$. Pharmacology interventions $(\mathrm{n}=3) ; 5$. Qualitative studies with no data available for meta-analysis $(\mathrm{n}=5) ; 6$. Interventions included were not technology-assisted $(n=33)$; and 7. Other reasons $(n=5)$
FIG. 1. Literature search flow diagram. 
Table 1. Descriptive Summary OF INCLUDED TRIALS $(N=28)$

\begin{tabular}{|c|c|c|}
\hline & $\begin{array}{l}\text { No. of } \\
\text { studies }\end{array}$ & Estimate \\
\hline \multicolumn{3}{|l|}{ Years of publication } \\
\hline Before 2000 & 1 & \\
\hline Between 2001 and 2010 & 6 & \\
\hline Between 2011 and 2015 & 12 & \\
\hline Between 2016 to present & 9 & \\
\hline Age of study participants (mean) & 28 & \\
\hline Age of study participants (range) & 28 & $4.2-32.17$ \\
\hline Gender ( $\%$ female $)$ & 27 & $45.86 \%$ \\
\hline \multicolumn{3}{|l|}{ Targeted population } \\
\hline Childhood cancer survivors only & 16 & $109 \mathrm{ES}$ \\
\hline AYA cancer survivors only & 9 & $100 \mathrm{ES}$ \\
\hline $\begin{array}{l}\text { Both childhood and AYA cancer } \\
\text { survivors }\end{array}$ & 3 & $28 \mathrm{ES}$ \\
\hline \multicolumn{3}{|l|}{ Targeted outcomes } \\
\hline $\begin{array}{l}\text { Distressful experience during } \\
\text { treatment }\end{array}$ & 5 & $25 \mathrm{ES}$ \\
\hline $\begin{array}{l}\text { Psychosocial and emotional } \\
\text { outcomes }\end{array}$ & 15 & $89 \mathrm{ES}$ \\
\hline Cancer related knowledge & 6 & $80 \mathrm{ES}$ \\
\hline Physical and functional health & 8 & $43 \mathrm{ES}$ \\
\hline \multicolumn{3}{|l|}{ Intervention modality } \\
\hline Individual-based intervention & 19 & \\
\hline Group-based intervention & 9 & \\
\hline \multicolumn{3}{|l|}{ Role of technology } \\
\hline $\begin{array}{l}\text { Technology has a primary role } \\
\text { in intervention }\end{array}$ & 22 & \\
\hline $\begin{array}{l}\text { Technology has an assistive role } \\
\text { in intervention }\end{array}$ & 6 & \\
\hline \multicolumn{3}{|l|}{ Involvement of human clinician } \\
\hline Primary provider of intervention & 13 & \\
\hline $\begin{array}{l}\text { Human clinician in a } \\
\text { secondary/assistive role }\end{array}$ & 15 & \\
\hline \multicolumn{3}{|l|}{ Technologies used } \\
\hline $\begin{array}{l}\text { Computer- or Tablet-based, } \\
\text { including DVD or Videos }\end{array}$ & 8 & \\
\hline Web-based, including Facebook & 4 & \\
\hline Interactive chatbot & 3 & \\
\hline (Educational) video games & 4 & \\
\hline Phone or text based & 4 & \\
\hline Virtual reality & 3 & \\
\hline Wearable (Fitbit) & 1 & \\
\hline $\begin{array}{l}\text { Multiple component } \\
\text { (web+phone+text message) }\end{array}$ & 1 & \\
\hline
\end{tabular}

AYA, adolescent and young adult; ES, effect sizes.

\section{Meta-analytic and subgroup analysis results}

Synthesizing across the 28 clinical trials (including the 237 effect sizes) yielded an overall treatment effect size for technology-assisted psychosocial interventions for childhood and AYA cancer survivors of $g=0.382,95 \%$ confidence interval (CI) (0.243 to 0.521), $p<0.0001$ (Table 3). Thus, childhood and AYA cancer survivors receiving technologyassisted interventions on average were expected to experience a 0.382 standard deviation greater improvement than their counterparts in the control condition. Subgroup analysis revealed that distraction-based interventions and interventions for psychosocial and emotional health reported overall

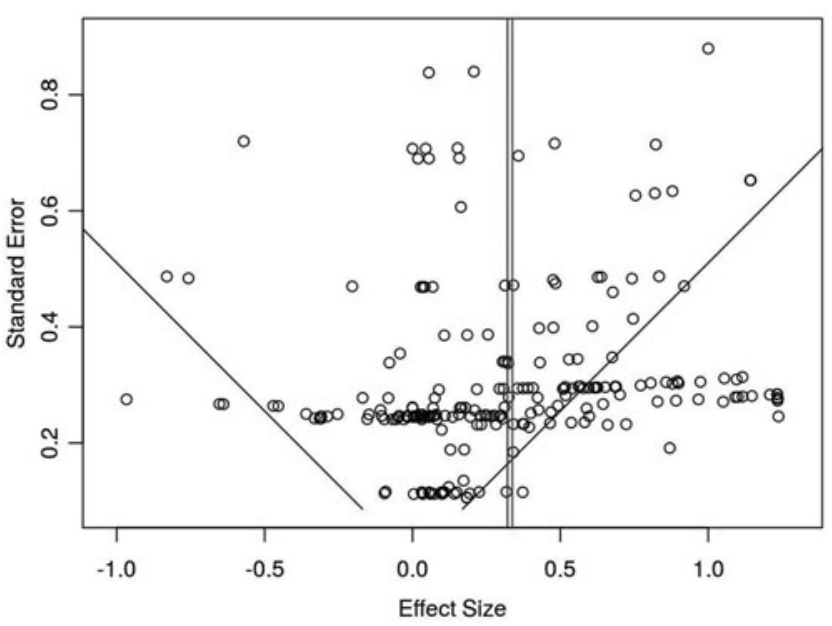

FIG. 2. Funnel plot for publication bias.

statistically significant treatment effects: $g=0.769,95 \% \mathrm{CI}$ (0.406 to 1.130$), p=0.0052$ and $g=0.299,95 \% \mathrm{CI}(0.105$ to $0.494), p=0.0060$, respectively. Technology-assisted interventions for childhood and AYA cancer survivors' cancer knowledge outcomes and physical and functional health outcomes were not statistically significant: $g=0.197,95 \% \mathrm{CI}$ (0.031 to 0.364$), p=0.0317$ and $g=0.269,95 \% \mathrm{CI}(0.014$ to $0.523), p=0.0416$, respectively.

Studies reported overall statistically significant treatment effects regardless of treatment modality, that is, in both individual- and group-based interventions: $g=0.315,95 \% \mathrm{CI}$ (0.134 to 0.497$), p=0.0023$ and $g=0.481,95 \% \mathrm{CI}(0.246$ to $0.716), p=0.0017$, respectively. Similarly, studies reported overall statistically significant treatment effects regardless of level of human involvement, that is, in those involving a human as a primary intervention provider and those involving a human for logistic/administrative check-in: $g=0.466,95 \%$ CI (0.244 to 0.688$), p=0.0007$ and $g=0.296,95 \%$ CI $(0.115$ to 0.478$), p=0.0043$, respectively.

Studies that used technology as the primary intervention or primary method of intervention delivery reported an overall statistically significant treatment effect: $g=0.419,95 \% \mathrm{CI}$ ( 0.255 to 0.583$), p<0.0001$. In contrast, studies that used technology as a complementary or assistive tool reported an overall treatment effect that was not statistically significant: $g=0.274,95 \%$ CI $(-0.104$ to 0.561$), p=0.1200$. The overall treatment effect of technology-assisted interventions delivered as psychotherapeutic treatments was not statistically significant: $g=0.455,95 \%$ CI $(-0.259$ to 1.170$), p=0.140$, whereas technology-assisted interventions delivered as nonpsychotherapeutic treatments (e.g., distraction or peer support) had an overall statistically significant treatment effect: $g=0.369,95 \%$ CI $(0.223$ to 0.515$), p<0.0001$.

Studies delivering technology-assisted interventions for childhood cancer survivors only had an overall statistically significant treatment effect: $g=0.414,95 \%$ CI $(0.184$ to $0.645), p=0.0019$. Studies delivering technology-assisted interventions for AYA cancer survivors only or for childhood and AYA cancer survivors had overall treatment effects that were not statistically significant: $g=0.283,95 \% \mathrm{CI}(0.053$ to $0.514), p=0.0229$ and $g=0.600,95 \%$ CI (0.214 to 0.986$)$, $p=0.0222$, respectively. 
Table 2. Risk of Bias for Randomized Controlled Trials and Controlled Trials Without Randomization

Randomized controlled trials

\begin{tabular}{|c|c|c|c|c|c|}
\hline Study citation & $B A R P$ & $B D I I$ & $B M O D$ & $B M O$ & $B S R R$ \\
\hline Akard et al. (2015) & + & + & $?$ & + & + \\
\hline Alemi et al. (2016) & $?$ & + & $?$ & + & + \\
\hline Beale et al. (2007) & $?$ & + & $?$ & + & + \\
\hline Burns et al. (2009) & $?$ & + & $\dot{+}$ & + & + \\
\hline Casillas et al. (2019) & + & + & $?$ & + & + \\
\hline Conklin et al. (2015) & + & + & $?$ & + & + \\
\hline Fazelniya et al. (2017) & + & + & $?$ & + & + \\
\hline Gershon et al. (2004) & + & + & $?$ & + & + \\
\hline Greer et al. (2019) & + & + & + & + & + \\
\hline Hardy et al. (2013) & $?$ & + & $?$ & + & + \\
\hline Howell et al. (2018) & $?$ & + & $?$ & + & + \\
\hline Huang et al. (2014) & + & + & + & + & + \\
\hline Jones et al. (2010) & $?$ & + & $?$ & + & + \\
\hline Kato et al. (2008) & + & + & + & + & + \\
\hline Klosky et al. (2004) & $?$ & + & $?$ & + & + \\
\hline Kunin-Batson et al. (2016) & $\dot{+}$ & + & $\dot{?}$ & + & + \\
\hline Mays et al. (2011) & $?$ & $?$ & + & + & + \\
\hline Mendoza et al. (2017) & $?$ & + & + & + & + \\
\hline Palmer et al. (2014) & $?$ & + & + & + & + \\
\hline Rabin et al. (2011) & $?$ & + & + & + & + \\
\hline Robb et al. (2014) & $?$ & + & + & + & + \\
\hline Sabel et al. (2016) & $?$ & + & $?$ & + & + \\
\hline Valle et al. (2013) & + & + & + & + & + \\
\hline Wolitzky et al. (2005) & $?$ & + & $?$ & + & + \\
\hline Wu et al. (2018) & $?$ & + & $?$ & + & + \\
\hline Yeh et al. (2011) & ? & + & $\dot{?}$ & + & + \\
\hline No. of studies (out of 26 studies) with low risk of bias & 10 & 25 & 10 & 26 & 26 \\
\hline No. of studies (out of 26 studies) with unsure/moderate risk of bias & 16 & 1 & 16 & 0 & 0 \\
\hline No. of studies (out of 26 studies) with serious or critical risk of bias & 0 & 0 & 0 & 0 & 0 \\
\hline
\end{tabular}

Controlled trials without randomization

\begin{tabular}{lcccccccc}
\hline & $B C$ & BSPS & BCI & BDII & BMOD & BMO & BSRR & OB \\
\hline Li et al. (2011) & + & + & + & + & $?$ & & + & + \\
Redd et al. (1987) & + & + & + & + & $?$ & & + & + \\
\hline
\end{tabular}

"+" = low risk of bias; "??" = unsure/moderate risk of bias.

BARP, bias arising from the randomization process; BC, bias due to confounding; BCI, bias in classification of interventions; BDII, bias due to deviations from intended interventions; BMO, bias in measurement of the outcome; BMOD, bias due to missing outcome data; BSPS, bias in selection of participants into the study; BSRR, bias in selection of the reported result; OB, overall bias.

\section{Moderator analysis}

The heterogeneity statistic, $Q(236)=647.44, p<0.0001$, indicated a significant amount of heterogeneity across effect size estimates, which warranted further moderator analyses (Table 4). Single-predictor univariate meta-regression analyses identified one significant moderator, intervention targets. When compared with distraction-based technologyassisted interventions, cancer knowledge outcomes $(b=-0.546$, $p=0.0065)$ and physical and functional health outcomes ( $b=-0.547, p=0.0045$ ) had significantly lower treatment effects, (i.e., these interventions were less effective). No other moderators evaluated were identified in this review.

\section{Discussion}

Overall, technology-assisted interventions are promising in supporting childhood and AYA cancer survivors' psychosocial health across multiple domains. In this systematic review and meta-analysis, we identified an overall statisti- cally significant treatment effect in relation to digital health interventions for childhood and AYA cancer survivors' distress and psychological wellness, but not for cancer-related knowledge or functional health outcomes. While the overall significant treatment effect was encouraging, oncologists and psycho-oncology providers need to critically evaluate available research evidence when considering technology-assisted interventions for different patient outcomes among pediatric and AYA cancer survivors. In other words, as indicated by the findings in this review, providers should not consider technology-assisted interventions effective for all outcome domains in pediatric and AYA cancer survivors, especially for improvements in cancer-related knowledge and functional health.

Digital health interventions that distract patients from invasive cancer treatments have received the strongest research support, evidenced by a statistically significant, large treatment effect. In addition, this study has shown that treatment effects for digital health interventions targeting childhood 
Table 3. Overall Treatment Effect and Subgroup Treatment Effects

\begin{tabular}{|c|c|c|c|c|c|}
\hline & Estimate & $K / N^{\mathrm{a}}$ & $d f s^{\mathrm{b}}$ & $95 \% C I$ & $\mathrm{p}$ \\
\hline Overall effect & 0.382 & $28 / 237$ & 23.4 & 0.243 to 0.521 & $<0.0001$ \\
\hline \multicolumn{6}{|l|}{ Intervention targets } \\
\hline Distraction of intrusive treatment & 0.769 & $5 / 25$ & 3.48 & 0.406 to 1.130 & 0.0052 \\
\hline Psychosocial and emotional health & 0.334 & $15 / 89$ & 12.5 & 0.120 to 0.547 & 0.0051 \\
\hline Cancer knowledge & 0.197 & $6 / 80$ & 3.39 & 0.031 to 0.364 & 0.0317 \\
\hline Physical and functional health & 0.269 & $8 / 43$ & 6.03 & 0.014 to 0.523 & 0.0416 \\
\hline \multicolumn{6}{|l|}{ Treatment modality } \\
\hline Individual based & 0.315 & $19 / 124$ & 14.1 & 0.134 to 0.497 & 0.0023 \\
\hline Other or mixed format & 0.481 & $9 / 113$ & 7.52 & 0.246 to 0.716 & 0.0017 \\
\hline \multicolumn{6}{|l|}{ Role of technology } \\
\hline Primary intervention & 0.419 & $22 / 178$ & 17.8 & 0.255 to 0.583 & $<0.0001$ \\
\hline Secondary assistive role & 0.274 & $6 / 59$ & 4.8 & -0.104 to 0.651 & 0.1200 \\
\hline \multicolumn{6}{|l|}{ Human clinician involvement } \\
\hline Human as primary provider & 0.466 & $13 / 157$ & 10.9 & 0.244 to 0.688 & 0.0007 \\
\hline Human provides logistic check-in & 0.296 & $15 / 80$ & 10.6 & 0.115 to 0.478 & 0.0043 \\
\hline \multicolumn{6}{|l|}{ Intervention nature } \\
\hline Therapeutic intervention & 0.455 & $5 / 31$ & 3.24 & -0.259 to 1.170 & 0.1400 \\
\hline Non-therapeutic intervention & 0.369 & $23 / 206$ & 19.5 & 0.223 to 0.515 & $<0.0001$ \\
\hline \multicolumn{6}{|l|}{ Patient group } \\
\hline Childhood cancer survivor only & 0.414 & $16 / 109$ & 12.8 & 0.184 to 0.645 & 0.0019 \\
\hline AYA cancer survivor only & 0.283 & $10 / 104$ & 6.89 & 0.053 to 0.514 & 0.0230 \\
\hline Childhood and AYA survivors & 0.600 & $3 / 28$ & 1.92 & 0.214 to 0.986 & 0.0222 \\
\hline
\end{tabular}

${ }^{\mathrm{a}} \mathrm{K}=$ number of studies; $N=$ number of effect size estimates.

b dfs, degrees of freedom. If $\mathrm{dfs}<4$, a stricter $p$-value of 0.01 should be used as threshold for statistical significance.

$\mathrm{CI}$, confidence interval.

and AYA cancer survivors' cancer knowledge and functional health are not significant. It may be that our findings on the lack of significant treatment effects of interventions targeting survivors' cancer knowledge were caused by the small number of studies involved, (i.e., evidenced by low degrees of freedom). However, our findings on digital health interventions for childhood and AYA survivors' functional health outcomes are consistent with a previous meta-analysis, which found that a distance-delivered physical activity intervention did not increase childhood cancer survivors' physical activity, but may improve their physical and functional health. ${ }^{28}$ Therefore, future research in survivorship care for childhood and AYA survivors should focus on the use of technologyassisted interventions in these areas and revisit the topics when more studies become available.

Furthermore, moderator analysis found that treatment effects for cancer knowledge and patients' functional health were both significantly smaller (i.e., less effective) than those for distraction-based technological interventions. One possible factor that may contribute to the difference in targeted outcomes (i.e., distraction-based interventions vs. knowl- edge or functional health outcomes) is the technology used. Many distraction-based interventions have employed advanced technologies such as virtual reality goggles or interactive chatbot, whereas interventions to improve cancer-related knowledge have employed psycho-educational computer programs or text messages as the primary technology. ${ }^{49}$ It is possible that virtual reality technologies are more engaging for childhood and AYA cancer survivors and that older technologies (e.g., text messaging) are less attractive among this population. As a result, the smaller treatment effects produced by these interventions are possibly caused by participants' poor engagement in these interventions. In addition, it is also possible that interventions with advanced technology are likely to have greater tailorability to patients' individual needs, whereas interventions targeting cancer knowledge or functional health are more structured and thus less suited to personalization. Future researchers should be mindful of developing and implementing engaging digital health interventions for childhood and AYA cancer survivors, especially those platforms that have a high potential for individualization and tailorability. ${ }^{50}$

Table 4. Univariate Meta-Regression Moderator Analysis

\begin{tabular}{lrrrrr}
\hline & Estimate & $K / N$ & $d f s^{\mathrm{a}}$ & $95 \%$ CI & $\mathrm{p}$ \\
\hline Intervention targets & & & & & \\
$\quad$ Distraction from intrusive treatment (ref.) & 0.766 & $28 / 237$ & 3.61 & 0.411 to 1.121 & 0.0047 \\
Psychosocial and emotional health & -0.367 & $28 / 237$ & 6.72 & -0.758 to 0.025 & 0.0623 \\
Cancer knowledge & -0.546 & $28 / 237$ & 6.89 & -0.882 to -0.209 & 0.0065 \\
Physical and functional health & -0.547 & $28 / 237$ & 8.77 & -0.875 to -0.219 & 0.0045 \\
\hline
\end{tabular}

${ }^{a} \mathrm{dfs}$, degrees of freedom.

ref., reference group. 
Treatment effects were statistically significant regardless of treatment modality (individual vs. nonindividual intervention) and the involvement of a human clinician (a human clinician as the primary interventionist vs. a human clinician providing logistic and supportive check-in). These findings are encouraging as they provide preliminary support for a variety of methods of delivering technology-assisted interventions to childhood and AYA cancer survivors and all can be equally effective. In contrast, however, only those interventions that used technology as the primary intervention (as opposed to using it in an assistive role) had statistically significant treatment effects. Studies included in this review used various technologies in supporting childhood and AYA cancer survivors ranging from text messages and Facebook chats (often in assistive roles) to virtual reality goggles and active video games (often as the primary intervention). It seemed that advanced technologies that are used as an independent intervention have greater potentials than using technologies in assistive roles for childhood and AYA cancer survivors.

The overall treatment effect of tech-based psychotherapeutic interventions was not statistically significant, which revealed an important gap in existing childhood and AYA cancer survivorship research. Psychotherapeutic interventions (such as cognitive behavioral therapy) are often delivered in a (semi)structured way to ensure treatment fidelity. When transferred to a technology-assisted platform, it is likely that some key components may be lost or insufficient during the process, which further compromises treatment fidelity and intervention effect. This suggests an important caveat for future psychosocial oncology studies: researchers must endeavor to ensure both engagement and fidelity when developing and implementing technology-assisted psychotherapeutic interventions to address psychological and emotional outcomes among childhood and AYA cancer survivors.

In addition, the treatment effect of technology-assisted interventions was only statistically significant across studies focused on pediatric cancer survivors and not across studies focused on AYA cancer survivors or childhood and AYA cancer survivors combined. In other words, technologyassisted interventions overall were not effective for AYA cancer survivors, a finding that is consistent with other studies. ${ }^{51}$ This may be perceived as a counterintuitive finding because AYAs, a tech-savvy generation, are often deemed "ideal candidates" for digital health interventions. One possible explanation for the discrepancy is that AYAs' appraisal of engaging technologies may be more demanding compared with their pediatric and adult counterparts. In other words, technology-based interventions that are considered engaging and appealing for pediatric and adult cancer survivors may be perceived as less attractive to AYA survivors, which could potentially contribute to a lower treatment effect (due to poor engagement). Also, it is also possible that AYA cancer survivors experience additional challenges and distress during their transition from pediatric to medical oncology. These challenges may make them less responsive to technologyassisted interventions, and many of these challenges require attention from health care systems and in health policies.

While advances in technology have a major potential to improve the accessibility of psychosocial interventions for all cancer survivors, including AYAs, investigators should carefully consider other key aspects of technology-assisted treatment development, namely treatment fidelity and treatments' interactivity and engagingness for AYAs. ${ }^{50}$ Investigators of technology-assisted interventions for AYA cancer survivors should adopt an interdisciplinary approach in developing technology-assisted platforms to improve interactivity and engagement, employing psycho-oncologists, computer scientists, media specialists, developmental psychologists, game designers, and specialists in other relevant disciplines. It is also essential for researchers and clinicians to incorporate the social determinants of health and health disparities in designing technology interventions supporting AYAs, given the unique transition-of-care challenges that are common among this population.

Finally, both demographic diversity among AYA cancer survivors and technology, information, and social media use in the lives of AYAs have been flagged as key components in developing a forward-looking approach to AYA psychooncology care. ${ }^{15}$ Therefore, the diversity among users and the plurality of contexts in which technologies are used must be critically incorporated into future studies about and implementation of technology-assisted psychosocial interventions for AYA cancer survivors. Innovative research approaches and clinical implementation strategies must account for health disparities associated with digital exclusion or "usage access gaps" that AYAs may face. ${ }^{52}$ Digital inequality is produced through variability in access to hardware and infrastructure, disparate digital skills, the differential usage/purpose of technology, and variable mental/emotional associations with technology use. Furthermore, demographic factors such as age, gender, race, and socioeconomic status influence the impact of these interrelated barriers (e.g., equipment and internet speed) on access to effective technology use. ${ }^{53}$ Therefore, demographic factors impacting digital inequality must be addressed in study design and service provision.

\section{Strengths and limitations}

A couple of limitations are worth noting. First, there is always a chance that the investigative team may have missed relevant articles, especially given that technology-assisted intervention studies may only include terms in their titles and abstracts that were not included in our search strategy. This concern, however, was largely alleviated after a thorough review of other existing systematic review and/or metaanalysis studies. Second, while our study used advanced statistical analysis to include multiple effect sizes from each study to improve statistical power, certain subgroup and moderator analyses still suffered from low statistical power (given the small sample size and the low number of effect sizes), which may have contributed to some findings that were not statistically significant. Third, our decision to use a wide age range as well as to include various forms of technology may confound some overall findings due to heterogeneity across studies. Therefore, it is important to interpret some of our overall findings in conjunction with findings from subgroup and moderator analyses. It is also worth noting that one included study (by Redd et al.) ${ }^{54}$ was published in 1987, while the rest were published in 2004 or later. Sensitivity analyses with and without inclusion of the study published in 1987 revealed no change; therefore, the Redd study was included. However, it is important to keep in mind 
that the use and definition of technology have changed drastically since 1987 , especially over the past 10 to 15 years. Finally, many included studies did not clearly differentiate between interventions that were delivered to childhood or AYA cancer survivors during treatment or after treatment (or both), which prevented us from distinguishing treatment effects of technology-assisted interventions for AYAs currently receiving treatment from treatment effects of those who completed therapy.

With that said, in this most recent and comprehensive systematic review and meta-analysis of technology-assisted interventions for childhood and AYA cancer survivors, we have not only used a comprehensive search strategy but also advanced statistical analyses that included 237 effect size estimates (across 28 clinical trials) for data synthesis. Given the substantially improved sample size of this review, both subgroup and moderator analyses were possible, which allowed a more nuanced interpretation of the existing literature on survivorship care for childhood and AYA cancer survivors. Given the comprehensiveness of this review, that is, the inclusion of a large number of studies and effect sizes, confidence was high on the findings we obtained.

\section{Conclusion}

This study showed that technology-assisted interventions for childhood and AYA cancer survivors were overall effective across domains of survivorship outcomes. Favorable evidence was found primarily for childhood cancer survivors with limited support for AYA cancer survivors. Based on the existing literature, there exists strong research evidence supporting technology-assisted interventions that distract childhood and AYA cancer survivors from intrusive cancer treatment and increase their psychosocial and emotional well-being. With recent advances in technology and the influence of social media, future studies need to focus on areas where evidence remains limited, that is, on areas related to survivors' cancer-related knowledge and the improvement of functional health outcomes.

\section{Acknowledgments}

Dr. Jessica Francis gave suggestions about relevant terminologies, citations, and concepts presented in the discussion section.

\section{Ethics Approval}

This is a meta-analysis that does not include sensitive or patient-identifying data; thus, IRB approval is not applicable.

\section{Disclaimer}

The content is solely the responsibility of the authors and does not necessarily represent the official views of the National Institutes of Health or the National Cancer Institute.

\section{Authors' Contributions}

A.Z. oversaw the entire study, screened and coded all studies, as well as conducted the second round of independent statistical analysis to ensure accuracy, and drafted the full article. B.Z. oversaw the entire study, coded some of the studies and resolved disagreement, and contributed the write- up of the article. C.A. and M.R. contributed to the write-up of the entire article. N.J.L. contributed to the discussion section of the study and proof read the entire study. K.W. conducted an independent, first round of statistical analysis. S.S. coded most of the studies and contributed to creating tables and figures.

\section{Author Disclosure Statement}

No competing financial interests exist.

\section{Funding Information}

N.J.L. received research support from the National Cancer Institute institutional training grant T32-CA-236621. A.Z. and N.J.L. received research support from the University of Michigan Vivian A. and James L. Curtis School of Social Work Center for Health Equity Research and Training, Signature Programs Initiatives.

\section{Supplementary Material}

Supplementary Appendix SA1

\section{References}

1. Johnston WT, Erdmann F, Newton R, et al. Childhood cancer: estimating regional and global incidence. Cancer Epidemiol. 2021;71(Pt B):101662.

2. Miller KD, Fidler-Benaoudia M, Keegan TH, et al. Cancer statistics for adolescents and young adults. CA Cancer J Clin. 2020;70(6):443-59.

3. Siegel RL, Miller KD, Jemal A. Cancer statistics, 2019. CA Cancer J Clin. 2019;69(1):7-34.

4. Baliga S, Yock TI. Pediatric cancer. Hematol Oncol Clin. 2020;34(1):143-159.

5. Trama A, Botta L, Steliarova-Foucher E. Cancer burden in adolescents and young adults: a review of epidemiological evidence. Cancer J. 2018;24(6):256-66.

6. Yeh JM, Ward ZJ, Chaudhry A, et al. Life expectancy of adult survivors of childhood cancer over 3 decades.. JAMA Oncol. 2020;6(3):350-57.

7. Scott AR, Stoltzfus KC, Tchelebi LT, et al. Trends in cancer incidence in US adolescents and young adults, 1973-2015. JAMA Netw Open. 2020;3(12):e2027738.

8. Naik H, Leung B, Laskin J, et al. Emotional distress and psychosocial needs in patients with breast cancer in British Columbia: younger versus older adults. Breast Cancer Res Treat. 2020;179(2):471-77.

9. Sender A, Friedrich M, Leuteritz K, et al. Unmet supportive care needs in young adult cancer patients: associations and changes over time. Results from the AYA-Leipzig study. J Cancer Surviv. 2019;13(4):611-9.

10. Stoneham SJ. AYA survivorship: the next challenge. Cancer. 2020;126(10):2116-9.

11. Rodgers C, Hooke MC, Ward J, Linder LA. Symptom clusters in children and adolescents with cancer. Semin Oncol Nurs. 2016;32(4):394-404.

12. Linder LA, Al-Qaaydeh S, Donaldson G. Symptom characteristics among hospitalized children and adolescents with cancer. Cancer Nurs. 2018;41(1):23-32.

13. Fidler MM, Frobisher C, Hawkins MM, Nathan PC. Challenges and opportunities in the care of survivors of adolescent and young adult cancers. Pediatr Blood Cancer. 2019;66(6):e27668. 
14. Barnett M, McDonnell G, DeRosa A, et al. Psychosocial outcomes and interventions among cancer survivors diagnosed during adolescence and young adulthood (AYA): a systematic review. J Cancer Surviv. 2016;10(5):814-31.

15. Levin NJ, Zebrack B, Cole SW. Psychosocial issues for adolescent and young adult cancer patients in a global context: a forward-looking approach. Pediatr Blood Cancer. 2019;66(8):e27789.

16. Lee JL, Gutierrez-Colina A, Williamson Lewis R, et al. Knowledge of late effects risks and healthcare responsibility in adolescents and young adults treated for childhood cancer. J Pediatr Psychol. 2019;44(8):557-66.

17. Psihogios AM, Schwartz LA, Deatrick JA, et al. Preferences for cancer survivorship care among adolescents and young adults who experienced healthcare transitions and their parents. J Cancer Surviv. 2019;13(4):620-31.

18. Szalda D, Piece L, Brumley L, et al. Associates of engagement in adult-oriented follow-up care for childhood cancer survivors. J Adolesc Health. 2017;60(2):147-53.

19. Tremolada M, Bonichini S, Basso G, Pillon M. Perceived social support and health-related quality of life in AYA cancer survivors and controls. Psychooncology. 2016; 25(12):1408-17.

20. Wettergren L, Kent EE, Mitchell SA, et al.; AYA HOPE Study Collaborative Group. Cancer negatively impacts on sexual function in adolescents and young adults: the AYA HOPE study. Psychooncology. 2017;26(10):1632-9.

21. Siembida EJ, Reeve BB, Zebrack BJ, et al. Measuring health-related quality of life in adolescent and young adult (AYA) cancer survivors with the NIH PROMIS ${ }^{\circledR}$ : comparing adolescent, emerging adult, and young adult survivor perspectives. Psychooncology. 2021;30(3):303-11.

22. Baird H, Patterson P, Medlow S, Allison KR. Understanding and improving survivorship care for adolescents and young adults with cancer. J Adolesc Young Adult Oncol. 2019;8(5):581-6.

23. Frederick NN, Bober SL, Berwick L, et al. Preparing childhood cancer survivors for transition to adult care: the young adult perspective. Pediatr Blood Cancer. 2017; 64(10):e26544.

24. Herts KL, Khaled MM, Stanton AL. Correlates of selfefficacy for disease management in adolescent/young adult cancer survivors: a systematic review. Health Psychol. 2017;36(3):192.

25. Wurz A, Brunet J. Describing and exploring self-esteem, physical self-perception, physical activity, and self-efficacy in adolescent and young adult cancer survivors. Eur $\mathrm{J}$ Cancer Care. 2020;29(1):e13179.

26. Zhou H, Xian W, Zhang Y, et al. Suicide among cancer patients: adolescents and young adult (AYA) versus all-age patients. Ann Transl Med. 2019;7(22):658.

27. Bukola IM, Paula D. The effectiveness of distraction as procedural pain management technique in pediatric oncology patients: a meta-analysis and systematic review. J Pain Symptom Manag. 2017;54(4):589-600.

28. Mizrahi D, Wakefield CE, Fardell JE, et al. Distancedelivered physical activity interventions for childhood cancer survivors: a systematic review and meta-analysis. Crit Rev Oncol Hematol. 2017;118:27-41.

29. Wender R. Integrating oncology, psychosocial, and medical care: the path forward. Psychooncology. 2020;29(3):461-4.

30. Grassi L, Fujisawa D, Odyio P, et al.; IPOS Federation of Psycho-oncology Societies' coauthors. Disparities in psychosocial cancer care: a report from the International
Federation of Psycho-oncology Societies. Psychooncology. 2016;25(10):1127-36.

31. Signorelli C, Wakefield CE, Fardell JE, et al. The impact of long-term follow-up care for childhood cancer survivors: a systematic review. Crit Rev Oncol Hematol. 2017;114: 131-8.

32. Doidge O, Edwards N, Thompson K, Lewin J. A conceptual framework to identify and address the education and vocational barriers experienced by adolescents and young adults with cancer. J Adolesc Young Adult Oncol. 2019; 8(4):398-401.

33. Hollis C, Falconer CJ, Martin JL, et al. Annual research review: digital health interventions for children and young people with mental health problems - a systematic and meta-review. J Child Psychol Psychiatry. 2017;58(4): 474-503.

34. Chan RJ, Howell D, Lustberg MB, et al. Advances and future directions in the use of mobile health in supportive cancer care: Proceedings of the 2019 MASCC Annual Meeting Symposium. Support Care Cancer. 2020;28(9):4059-67.

35. Janssen A, Brunner M, Keep $\mathbf{M}$, et al. Interdisciplinary eHealth practice in cancer care: a review of the literature. Int J Environ Res Public Health. 2017;14(11):1289.

36. Mendoza JA, Baker KS, Moreno MA, et al. A Fitbit and Facebook mHealth intervention for promoting physical activity among adolescent and young adult childhood cancer survivors: a pilot study. Pediatr Blood Cancer. 2017;64(12):e26660.

37. Psihogios AM, Li Y, Butler E, et al. Text message responsivity in a 2-way short message service pilot intervention with adolescent and young adult survivors of cancer. JMIR Mhealth Uhealth. 2019;7(4):e12547.

38. Hill-Kayser CE, Szalda D, Vachani C, et al. Feasibility and acceptability of survivorship care plans for adolescent/ young adult survivors of childhood cancer. Int $\mathbf{J}$ Radiat Oncol Biol Phys. 2019;105(1):e602-3.

39. Devine KA, Viola AS, Coups EJ, Wu YP. Digital health interventions for adolescent and young adult cancer survivors. JCO Clin Cancer Inform. 2018;2:1-15.

40. Ramsey WA, Heidelberg RE, Gilbert AM, et al. eHealth and mHealth interventions in pediatric cancer: a systematic review of interventions across the cancer continuum. Psychooncology. 2020;29(1):17-37.

41. Mayer DK, Nasso SF, Earp J. A. Defining cancer survivors, their needs, and perspectives on survivorship health care in the USA. Lancet Oncol. 2017;18(1):e11-8.

42. Shamseer L, Moher D, Clarke M, et al. A. Preferred Reporting Items for Systematic Review and Meta-Analysis Protocols (PRISMA-P) 2015: elaboration and explanation. BMJ. 2015:350:g7647.

43. Sterne JA, Savović J, Page MJ, et al. RoB 2: a revised tool for assessing risk of bias in randomised trials. BMJ. 2019; 366:14898.

44. Jüni P, Loke Y, Pigott T, et al. Risk of Bias in Nonrandomized Studies of Interventions (ROBINS-I): detailed guidance. BMJ. 2016;355:i4919.

45. Vevea JL, Woods CM. Publication bias in research synthesis: sensitivity analysis using a priori weight functions. Psychol Methods. 2005;10(4):428.

46. Cooper H, Hedges LV, Valentine JC. The handbook of research synthesis and meta-analysis. New York, NY: Russell Sage Foundation; 2019.

47. Hedges LV, Tipton E, Johnson MC. Robust variance estimation in meta-regression with dependent effect size estimates. Res Synth Methods. 2010;1(1):39-65. 
48. Moeyaert M, Ugille M, Natasha Beretvas S, et al. Methods for dealing with multiple outcomes in meta-analysis: a comparison between averaging effect sizes, robust variance estimation and multilevel meta-analysis. Int $\mathbf{J}$ Soc Res Methodol. 2017;20(6):559-72.

49. Smith AB, Bashore L. The effect of clinic-based health promotion education on perceived health status and health promotion behaviors of adolescent and young adult cancer survivors. J Pediatr Oncol Nurs. 2006;23(6):326-34.

50. Himle JA, Weaver A, Zhang A, Xiang X. Digital mental health interventions for depression. Cogn Behav Pract. 2021; DOI: 10.1016/j.cbpra.2020.12.009.

51. Zhang A, Wang K, Zebrack B, et al. Psychosocial, behavioral, and supportive interventions for pediatric, adolescent, and young adult cancer survivors: a systematic review and meta-analysis. Crit Rev Oncol Hematol. 2021; 160:103291.

52. Ball C, Huang KT, Francis J, et al. A call for computer recess: the impact of computer activities on predominantly minority students' technology and application self-efficacy. Am Behav Sci. 2020;64:883-99.
53. Francis J, Ball C, Kadylak T, Cotten SR. Aging in the digital age: conceptualizing technology adoption and digital inequalities. In: Neves B, Vetere F (Eds). Ageing and Digital Technology. Singapore: Springer; 2019. https://doi .org/10.1007/978-981-13-3693-5_3

54. Redd WH, Jacobsen PB, Die-Trill M, et al. Cognitive/ attentional distraction in the control of conditioned nausea in pediatric cancer patients receiving chemotherapy. J Consult Clin Psychol. 1987;55(3):391.

Address correspondence to: Anao Zhang, PhD, MSW University of Michigan School of Social Work 1080 S. University Ave. Ann Arbor, MI 48109

USA

Email: zhangan@umich.edu 